\title{
Adiponectin improves insulin sensitivity via activation of autophagic flux
}

\author{
Penny Ahlstrom, Esther Rai, Suharto Chakma, Hee Ho Cho, Palanivel Rengasamy \\ and Gary Sweeney
}

Department of Biology, York University, Toronto, Canada
Correspondence should be addressed to G Sweeney

Email

gsweeney@yorku.ca

\begin{abstract}
Skeletal muscle insulin resistance is known to play an important role in the pathogenesis of diabetes, and one potential causative cellular mechanism is endoplasmic reticulum (ER) stress. Adiponectin mediates anti-diabetic effects via direct metabolic actions and by improving insulin sensitivity, and we recently demonstrated an important role in stimulation of autophagy by adiponectin. However, there is limited knowledge on crosstalk between autophagy and ER stress in skeletal muscle and in particular how they are regulated by adiponectin. Here, we utilized the model of high insulin/glucose (HIHG)-induced insulin resistance, determined by measuring Akt phosphorylation (T308 and S473) and glucose uptake in L6 skeletal muscle cells. HIHG reduced autophagic flux measured by LC3 and p62 Western blotting and tandem fluorescent RFP/GFP-LC3 immunofluorescence (IF). HIHG also induced ER stress assessed by thioflavin T/KDEL IF, pIRE1, pPERK, peIF2 $\alpha$ and ATF6 Western blotting and induction of a GRP78-mCherry reporter. Induction of autophagy by adiponectin or rapamycin attenuated HIHG-induced ER stress and improved insulin sensitivity. The functional significance of enhanced autophagy was validated by demonstrating a lack of improved insulin sensitivity in response to adiponectin in autophagy-deficient cells generated by overexpression of dominant negative mutant of Atg5. In summary, adiponectin-induced autophagy in skeletal muscle cells alleviated HIHG-induced ER stress and insulin resistance.
\end{abstract}

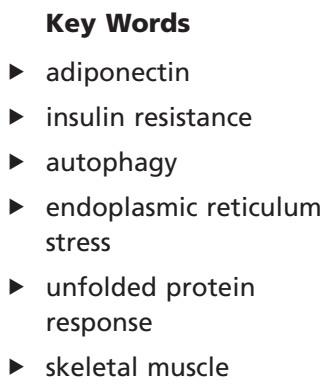

Journal of Molecular Endocrinology (2017) 59, 339-350

\section{Introduction}

Diabetes currently affects over 422 million people worldwide and type 2 , the most common form, arises from an initial impairment of peripheral tissue insulin sensitivity. Skeletal muscle is responsible for clearing the majority of circulating glucose in response to insulin, and it is therefore critical to fully understand the mechanisms leading to insulin resistance and how they can be alleviated (Marette et al. 2014). A strong inverse correlation of circulating adiponectin with diabetes and skeletal muscle insulin resistance exists in humans and animal models (Stern et al. 2016). Adiponectin is now established as an anti-diabetic hormone (Cheng et al. 2014). Muscle is one important target tissue of adiponectin, and we have reviewed the metabolic actions of adiponectin in skeletal muscle and the mechanisms via which these have been shown to occur (Liu \& Sweeney 2014). Importantly, we recently demonstrated that adiponectin stimulates autophagic flux in skeletal muscle and that increased autophagy contributed to insulin-sensitizing anti-diabetic effects (Liu et al. 2015, Xu \& Sweeney 2015). Others have now also shown that boosting autophagy via a variety of approaches can enhance insulin sensitivity in 
skeletal muscle and other tissues (Wang et al. 2015, Zhu et al. 2016, Ghareghani et al. 2017, Li et al. 2017, RosaCaldwell et al. 2017) and vice versa that decreased the levels of skeletal muscle autophagy were observed in type 2 diabetes and upon aging (Zhang et al. 2016, Moller et al. 2017, Zhou et al. 2017).

Until recently, the causative role of ER stress in promoting insulin resistance in skeletal muscle was much less studied than other tissues. However, there is now a strong body of evidence, which clearly indicates that various factors can elevate ER stress in skeletal muscle leading to insulin resistance. For example, inflammation or high-fat diet can lead to ER stress (Dai et al. 2016, Kwak et al. 2016, Liong \& Lappas 2016). Various reports have demonstrated that treatments which alleviate skeletal muscle ER stress induced by stressors such as highfat diet or palmitate are able to elicit a corresponding improvement in insulin sensitivity (Hwang et al. 2013, Salvado et al. 2014, Quan et al. 2015, Dai et al. 2016, Kwak et al. 2016). To further elucidate the mechanisms responsible for adiponectin's insulin-sensitizing effect on skeletal muscle, additional research is needed to examine crosstalk between autophagy and ER stress.

We hypothesized that the interplay between adiponectin-stimulated autophagy and ER stress plays an important role in the regulation of skeletal muscle insulin sensitivity. To investigate this, we used an established cellular model of skeletal muscle insulin resistance, namely high insulin high glucose (HIHG) treatment in L6 cells (Huang et al. 2002) treated with or without adiponectin. We also generated an autophagy-deficient L6 cell line by stably overexpressing a dominant negative point mutant of Atg5 to allow examination of the functional significance of autophagy during the development of insulin resistance.

\section{Materials and methods}

\section{Cell culture, stable cell lines and cellular model of insulin resistance}

L6 myoblasts were maintained in alpha-minimum essential medium (AMEM; $5.5 \mathrm{mM}$ glucose) supplemented with $10 \%$ fetal bovine serum (FBS) in $75 \mathrm{~cm}^{2}$ flasks at $37^{\circ} \mathrm{C}$ in $5 \% \mathrm{CO}_{2}$. Differentiation from myoblasts to myotubes was induced by serum starvation in 2\% FBS AMEM for 6 days. Prior to treatments, cells were starved for $3 \mathrm{~h}$ in $0.5 \%$ FBS AMEM with relevant chemical added as indicated in figure legends after $2 \mathrm{~h}$ of starvation, thus allowing a $1 \mathrm{~h}$ pre-treatment before experimental starting point. AMEM used for growing stable cell lines contained puromycin to select for transgene-expressing cells. Insulin resistance was induced by incubating cells in high insulin (HI; $100 \mathrm{nM}$ Humulin R, purchased from Eli Lilly and Company) and high glucose (HG; $25 \mathrm{mM}$ D-glucose purchased from Sigma-Aldrich) containing AMEM with $0.5 \%$ FBS (HIHG) for $24 \mathrm{~h}$. A stable L6 cell line transfected to overexpress GLUT4 (a kind gift from Dr Amira Klip, The Hospital for Sick Children, Toronto) was used to assess insulin-stimulated glucose uptake in myoblasts (Huang et al. 2002).

A stable L6 GRP78mCherry reporter cell line containing the GRP78 promoter controlling the expression of mCherry, allowing the fluorescent signal to be used as visual readout of GRP78 gene expression, was generated by retroviral infection. The GRP78mCherry target vector (a kind gift from Dr Allen Volchuk, Toronto General Research Institute/University Health Network, Canada) was incorporated into the retroviral cloning vector pQCXIP by restriction and ligation. Once purified, the viral vector was transfected into EcoPack 2-293 (Clontech Laboratories). This human embryonic kidney-derived packaging cell line express Moloney murine leukemia virus Gag, Pol and Env proteins. $48 \mathrm{~h}$ after transfection the culture media containing the virus was collected and $100 \mu \mathrm{L}$ of the supernatant was added together with polybrene $(4 \mu \mathrm{g} / \mathrm{mL})$ to L6 cells 1 day after being seeded in $10-\mathrm{cm}$ culture dishes. After being incubated with the virus for $24 \mathrm{~h}$ the growth media was replaced with fresh growth media containing puromycin $(2 \mu \mathrm{g} / \mathrm{mL}$; Sigma-Aldrich) as the selection antibiotic and the stable expression of mCherry was verified by FACSCalibur flow cytometry (BD bioscience) (Lai et al. 2008, Liu et al. 2015). L6 cells stably expressing tandem fluorescent LC3 (TFLC3) was generated as we described before (Liu et al. 2015). A stable L6 cell line overexpressing an Atg5-dominant negative mutant (Atg5K130R (Atg5K)), which prevents conjugation to ATG12 and blocks LC3II incorporation and elongation of the autophagosome membrane was generated as previously described by us (Liu et al. 2015). Cells expressing empty vector (EV) were used as control.

\section{Materials}

Adiponectin was produced as we previously described (Palanivel et al. 2007) and dissolved in phosphate-buffered saline (PBS), and then media for use at $5 \mu \mathrm{g} / \mathrm{mL}$. All culture media and solutions were purchased from Wisent (SaintJean-Baptiste, QC, Canada). Tunicamycin (Sigma-Aldrich) was dissolved in DMSO to a stock concentration of $5 \mathrm{mM}$.

Published by Bioscientifica Ltd. 
Rapamycin (Sigma-Aldrich) was dissolved in PBS to a stock concentration of $27.4 \mu \mathrm{M}$. Bafilomycin A1 (SigmaAldrich) was dissolved in DMSO to a stock concentration of $100 \mu \mathrm{M}$.

\section{Western blot analysis}

Upon completion of treatment, L6 cells were lysed in RIPA lysis buffer $(50 \mathrm{mM}$ Tris, $150 \mathrm{mM} \mathrm{NaCl}, 0.1 \%$ SDS, $1 \%$ Triton $\mathrm{X}$ and $0.5 \%$ sodium deoxycholate) containing $10 \%$ $\beta$-mercaptoethanol and phosphatase inhibitor cocktail set $\mathrm{V}$ (EMD Millipore), heated for $10 \mathrm{~min}$ in $90^{\circ} \mathrm{C}$ and centrifuged at $13,600 \boldsymbol{g}$ for up to $15 \mathrm{~min}$. Samples were resolved by $8-15 \%$ sodium dodecyl sulfate polyacrylamide gel electrophoresis (SDS-PAGE). Proteins were transferred onto a polyvinylidene fluoride (PVDF) membrane, blocked in 3\% bovine serum albumin (BSA) blocking buffer and then immunoblotted with primary antibodies (pPERK, peIf $2 \alpha$, LC3, pAKT(T308), pAKT(S473), $\beta$-actin and GAPDH purchased from Cell Signaling, pIRE1 purchased from Novus Biologicals, ATF6 purchased from Santa Cruz, p62 antibody from BD Biosciences (Mississauga, ON, Canada) and KDEL purchased from Abcam. These were subsequently detected with horseradish peroxidase (HRP)-conjugated secondary antibodies purchased from Cell Signaling. Protein bands were visualized using enhanced chemiluminescence (ECL, BioRad) reagent and quantitated by densitometry using ImageJ. All values were corrected for an appropriate loading control, $\beta$-actin or GAPDH.

\section{Glucose uptake}

Glucose uptake was assessed exactly as previously described (Liu \& Sweeney 2014).

\section{Thioflavin T immunofluorescence and KDEL colocalization}

Thioflavin T (ThT) dye exhibits increased fluorescence when bound by protein aggregates such that the level of fluorescence has been shown to correlate with ER stress inducers (Beriault \& Werstuck 2013). Here, ThT dye was added to live cells for $40 \mathrm{~min}(1 \mu \mathrm{M} / \mathrm{mL})$, media was aspirated and cells were washed before fixing in $4 \%$ paraformaldehyde for 20 min. Coverslips were then mounted on slides in Vectashield mounting media containing DAPI. When ThT assay was combined with KDEL immunofluorescence, after fixing, the cells were quenched in $0.1 \%$ glycine in PBS for $10 \mathrm{~min}$, washed with PBS and permeabilized for 3 min in $0.1 \%$ Triton $X 100$, followed by blocking for $30 \mathrm{~min}$ in $3 \% \mathrm{BSA}$, and then incubated overnight at $4^{\circ} \mathrm{C}$ with $1: 500$ primary KDEL antibody. Cells were then washed and subsequently incubated with 1:1000 secondary antibody Alexa Fluor 594 for $1 \mathrm{~h}$ in the dark at room temperature and washed prior to mounting on slides in DAKO mounting media. Slides were imaged with Zeiss LSM 700 Confocal Microscope. Average fluorescence per cell was quantified using Zen 2012 Blue Edition, and Manders Coefficient analysis of colocalization of ThT (green) to KDEL (red) fluorescence was assessed using ImageJ software (JaCoP plugin).

\section{GRP78-mCherry reporter assay}

After treatment, cells were fixed in 4\% paraformaldehyde for 20 min, washed 3 times in cold PBS and mounted on slides in DAKO mounting media. Stacked images (12 slices) were taken with Zeiss LSM 700 Confocal Microscope and images quantified using ImageJ software as average fluorescence intensity per cell for 40 cells/treatment/ replicate experiment; all other images were taken as single images.

\section{Tandem fluorescent RFP/GFP-LC3 assay}

After treatment cells were fixed in 4\% paraformaldehyde for $20 \mathrm{~min}$, washed 3 times in cold PBS and mounted on slides in DAKO mounting media. To assess autophagosome formation, green and red merged images were converted to 8-bit black and white images and number of puncta counted using ImageJ Particle Analysis. To measure autophagic flux, images were quantified as total red/green fluorescence intensity per image for 8-10 images using Zen 2012 Blue Edition.

\section{Statistical analysis}

Comparison between two groups were done using unpaired one-tailed $t$-test and comparisons between multiple groups were done by one-way ANOVA, where data was found to not be normally distributed the nonparametric equivalents were used (Mann-Whitney $U$ and Kruskal-Wallis, respectively). Significant ANOVAs were followed by Tukey's HSD post hoc test when differences between all groups were of interest and Dunnett's post hoc test when all groups were compared to control. $P<0.05$ was accepted as significant. http://jme.endocrinology-journals.org DOI: 10.1530/JME-17-0096
() 2017 Society for Endocrinology Printed in Great Britain
Published by Bioscientifica Ltd 


\section{Results}

\section{HIHG treatment induced insulin resistance in L6 skeletal muscle cells}

Insulin-stimulated glucose uptake was attenuated after 24-h HIHG treatment, although HIHG itself increased basal transport in L6 myotubes (Fig. 1A). HIHG-induced insulin resistance in L6 myotubes was also confirmed by examining the level of AKT phosphorylation in response to $100 \mathrm{nM}$ of insulin at $0,1,3,6,8$ and $10 \mathrm{~min}$ by Western blot. Insulin increased AKT phosphorylation at both the threonine 308 and serine 473 phosphorylation sites, whereas 24-h HIHG treatment clearly attenuated this response (Fig. 1B). We confirmed that there was no significant change in the total Akt protein expression after 24-h HIHG treatment (Fig. 1C). The insulinsensitizing ability of adiponectin was then assessed with or without 24-h HIHG treatment. Results in Fig. 1D examining Akt phosphorylation on T308 demonstrated that adiponectin significantly attenuated HIHG-induced insulin resistance. Together, these data establish the cellular model of HIHG-induced insulin resistance and the beneficial effect of adiponectin in maintaining insulin sensitivity.

\section{HIHG attenuated autophagic flux}

In order to examine alterations in autophagy and their functional significance, we first treated cells with
HIHG for 2, 6 and $24 \mathrm{~h}$ and confirmed reduced insulinstimulated AKT phosphorylation on T308 at all times, with $24 \mathrm{~h}$ having the biggest effect (Fig. 2A). HIHG also decreased content of the autophagosome marker protein LC3II at each time point (Fig. 2B). Bafilomycin A (BafA), an inhibitor of autophagosome lysosome fusion, was used here as a positive control to attenuate autophagic flux and thus elicit an accumulation of LC3II. We also observed by Western blotting that p62 levels accumulated after HIHG treatment (Fig. 2C). We observed that 24-h HIHG treatment reduced LC3-II levels (Fig. 2D). Tandem fluorescence LC3 (TFLC3) expressing cells were then used here to monitor the process of autophagic flux in more detail. This assay is based on the fact that the acidic environment in the lysosome quenches green but not red fluorescence, thus, a higher red/green fluorescence ratio indicates more autophagic flux. We observed a reduced red/ green fluorescence ratio observed following BafA treatment, indicating less autophagosome fusion with lysosomes and also observed that HIHG had a similar effect in reducing autophagic flux (Fig. 2E). To further examine the inhibitory effect of HIHG on autophagic flux, we examined the number of fluorescent 'puncta' using ImageJ particle analysis (Fig. 2F) demonstrating an expected elevation in LC3-positive puncta in BafAtreated cells but with a reduced number of LC3-positive puncta after HIHG, suggesting that HIHG attenuates the early stage of autophagy initiation.
A

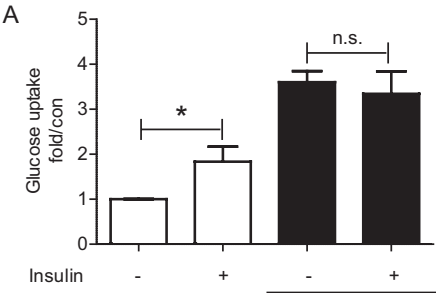

C

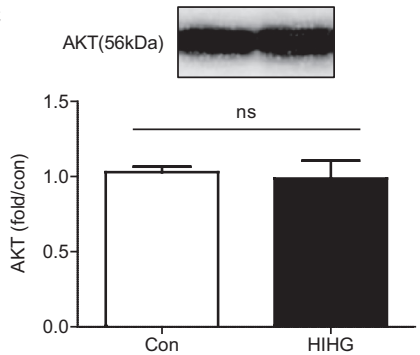

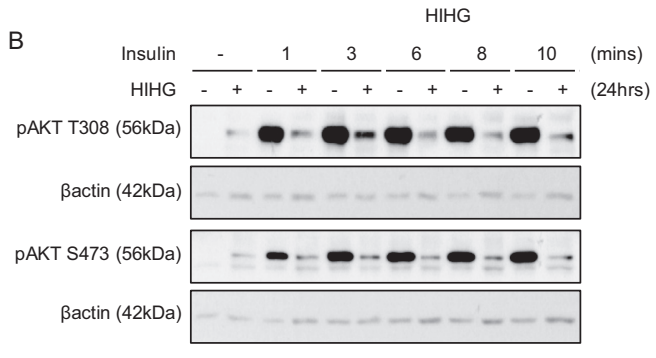

D

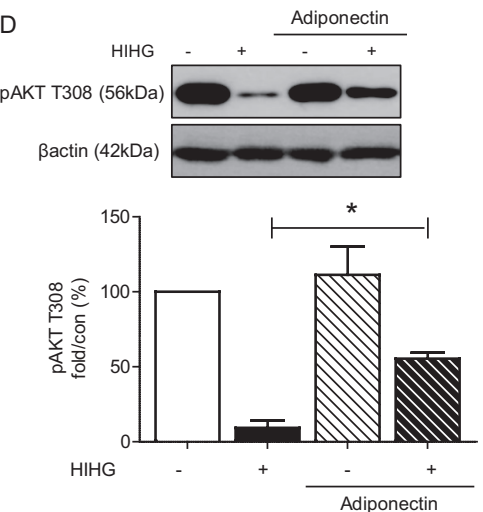

Figure 1

Skeletal muscle insulin resistance was induced by exposing L6 cells to HIHG condition and reversed by adiponectin treatment. (A) Insulin (100 nM) stimulated $(3 \mathrm{H}) 2$-deoxy-d-glucose uptake with or without 24-h HIHG treatment in L6 skeletal muscle cells; (B) Representative images of AKT phosphorylation in control or HIHG condition in response to $0,1,3,6,8$ and $10 \mathrm{~min}$ of $100 \mathrm{nM}$ insulin stimulation; (C) Representative image of total AKT in control or HIHG condition. (D) Representative images and densitometry analysis of $5 \mathrm{~min} 100 \mathrm{nM}$ insulin-stimulated AKT phosphorylation upon HIHG treatment with or without adiponectin pre-treatment. $n=3$ for each experiment. Values are mean \pm S.E.M., ${ }^{\star} P<0.05$. 

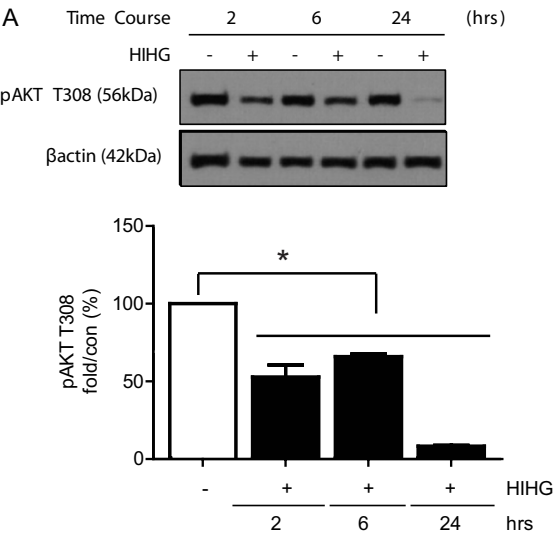

C

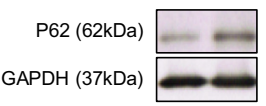

D

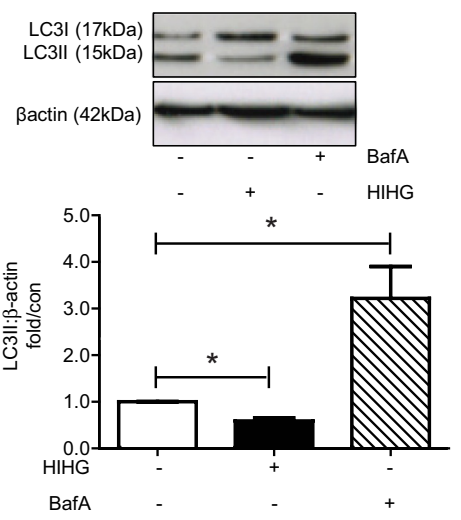

$\mathrm{F}$

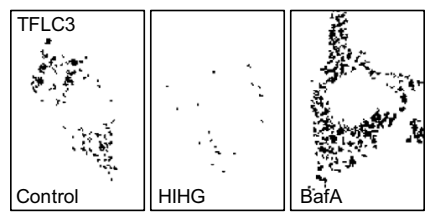

B
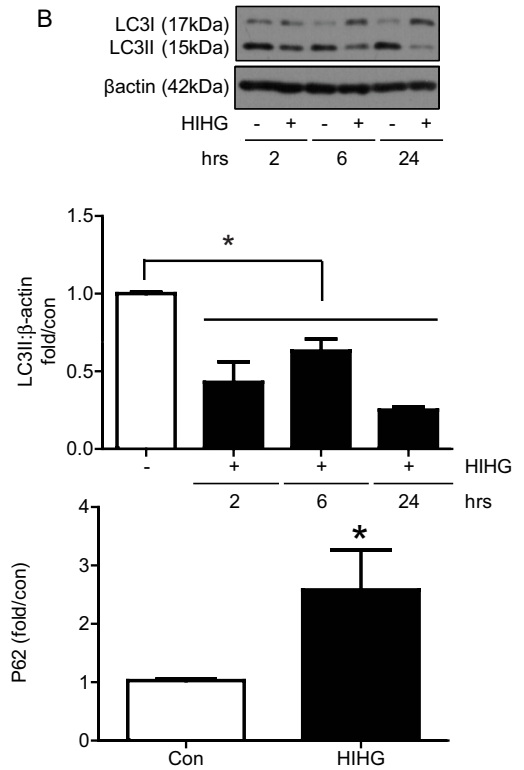

E
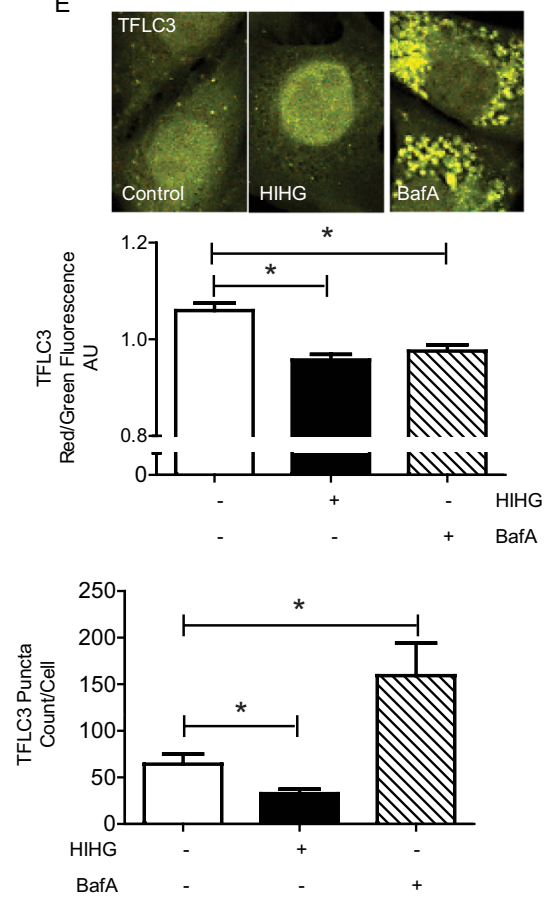

Figure 2

Insulin resistance in skeletal muscle cells was associated with impairment in autophagy. (A) Representative images and densitometry analysis of $5 \mathrm{~min} 100 \mathrm{nM}$ insulin-stimulated AKT phosphorylation upon 2-, 6- and 24-h HIHG treatment, $n=4$; (B) Representative images and densitometry analysis of LC3 in HIHG condition for $0,2,6$, and 24h, $n=4$; (C) Representative images and densiometry analysis of P62 in $24 \mathrm{~h}$ of HIHG treatment compared to control, $n=6$. (D) Representative images and densitometry analysis of LC3 in $24 \mathrm{~h}$ HIHG treatment compared to BafA-treated L6 muscle cells, $n=4$;

(E) Representative confocal images of tandem fluorescence-GFP-RFP-LC3 L6 cells and quantitative statistical analysis of red-to-green fluorescence ratio in HIHG or BafA-treated cells, $n=7$; (F) Representative images and quantification of puncta using ImageJ Particle Analysis with or without HIHG or BafA treatment, $n=7$. Values are mean \pm S.E.M., $* P<0.05$. A full color version of this figure is available at http:// dx.doi.org/10.1530/JME-17-0096.

\section{HIHG elevated ER stress and UPR activation}

We next performed a series of experiments to examine the effect of HIHG on ER stress and related unfolded protein response (UPR) signaling pathway in skeletal muscle cells. Thioflavin $\mathrm{T}$ (ThT) was used to visualize misfolded protein aggregates and thus quantify levels of ER stress and indicated a small but significant increase in ER stress after 24, but not 4, hours of HIHG treatment (Fig. 3A). Increased ER stress was accompanied by an increase in UPR activation, determined by transfecting
L6 cells with a GRP78mCherry reporter. The increased level of mCherry fluorescence in cells treated with HIHG for $24 \mathrm{~h}$ compared to control cells indicated an increase in UPR activation (Fig. 3B). Tunicamycin, a specific inhibitor of N-linked glycosylation, was used here as a positive control for the activation of ER stress in both assays (Fig. 3A and B). Further analysis of UPR pathways was examined by Western blot. Figure 3C demonstrated that HIHG significantly increased phosphorylation of 

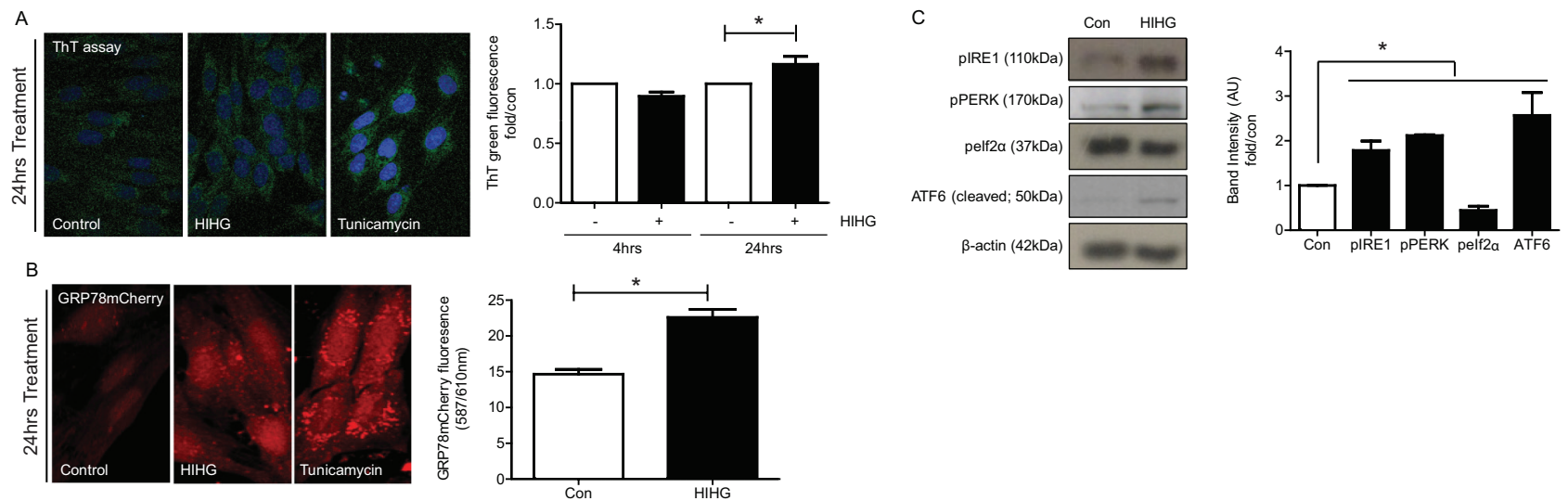

Figure 3

ER stress and UPR activation was increased in insulin-resistant skeletal muscle cells. (A) ThT assay measuring ER stress with representative confocal images and quantitative and statistical analysis of total green fluorescence per cell for 4 and $24 \mathrm{~h}$ HIHG-treated cells compared to control, $n=3$. Tunicamycin was used here as positive control to induce ER stress; (B) Representative confocal images of L6 GRP78mCherry UPR-reporter and statistical analysis of mean red fluorescence per cell for HIHG condition compared to control, $n=7$. Tunicamycin was used here as positive control to induce ER stress;

(C) Representative images and densitometry analysis of the UPR markers pIRE1, pPERK, pelf $2 \alpha$ and ATF6 in HIHG condition compared to control, $n=4$. Values are mean \pm S.E.M., ${ }^{*} P<0.05$. A full color version of this figure is available at http://dx.doi.org/10.1530/JME-17-0096.

IRE1, PERK and the cleaved form of ATF6. However, an unexpected decrease in phosphorylation of pelf $2 \alpha$ was observed (Fig. 3C).

\section{Rapamycin reduced HIHG-induced ER stress in an autophagy-dependent manner}

In order to study the inter-relationship between ER stress and autophagy in response to HIHG treatment in skeletal muscle cells, we first used a gain of function approach with rapamycin as a well-established inducer of autophagy. Again using TFLC3 assay, rapamycin treatment significantly induced autophagic flux and restored the impairment in autophagy caused by HIHG (Fig. 4A). This ability of rapamycin to induce autophagy and recover HIHG-impaired autophagy was also confirmed by Western blotting analysis of LC3II levels (Fig. 4B). We then used a loss-of-function approach by generating an autophagy-deficient cell line (Atg5K130R cells). As expected, the ability of rapamycin to induce autophagy \pm HIHG was absent in Atg5K130R cells (Fig. 4B). Having characterized the approaches for gain or loss of function of autophagy, we then used them to examine HIHG-induced ER stress. ThT assay demonstrated that rapamycin significantly reduced HIHG-induced ER stress (Fig. 4C). However, this effect of rapamycin was lost in autophagy-deficient cells (Fig. 4C). These data indicate that targeting autophagy is an effective means to reduce HIHG-induced ER stress in skeletal muscle cells.
Rapamycin increased autophagy and attenuated HIHG-induced insulin resistance in control but not autophagy-deficient cells

The importance of autophagy in determining insulin sensitivity was investigated and AKT phosphorylation (S473) data showing that both HIHG and BafA induced insulin resistance, which could suggest that reduced autophagy correlated with reduced insulin sensitivity (Fig. 5A). Both HIHG and BafA increased ER stress, indicated by ThT fluorescence (Fig. 5B). Next, the effect of bafilomycin or rapamycin on insulin sensitivity was examined in both EV and Atg5K cells. Rapamycin directly enhanced insulin sensitivity, and most importantly, we observed that rapamycin also significantly improved HIHG-induced insulin resistance in normal but not autophagy-deficient cells (Fig. 5C). Bafilomycin directly reduced insulin sensitivity and also exacerbated HIHGinduced insulin resistance (Fig. 5C).

\section{Adiponectin alleviated HIHG-induced ER stress and insulin resistance in an autophagy-dependent manner}

Adiponectin has been shown previously to stimulate autophagy and here we observed that adiponectin prevented the HIHG-induced decrease in LC3II (Fig. 6A). TFLC3 assay red/green fluorescence (Fig. 6B) and TFLC3 puncta analysis (Fig. 6C) also indicated that adiponectin prevented the HIHG-induced decrease in autophagic flux. Furthermore, adiponectin alleviated HIHG-induced

Published by Bioscientifica Ltd 
A

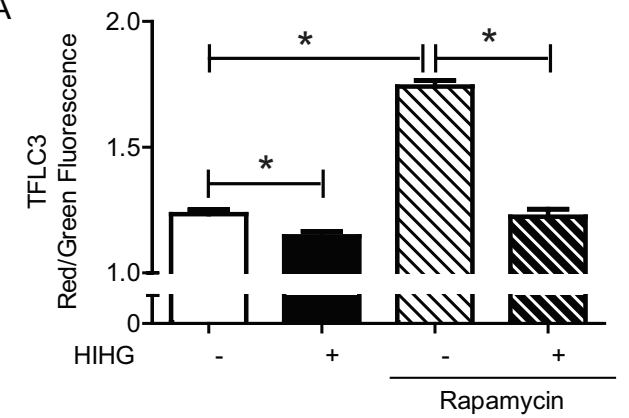

B
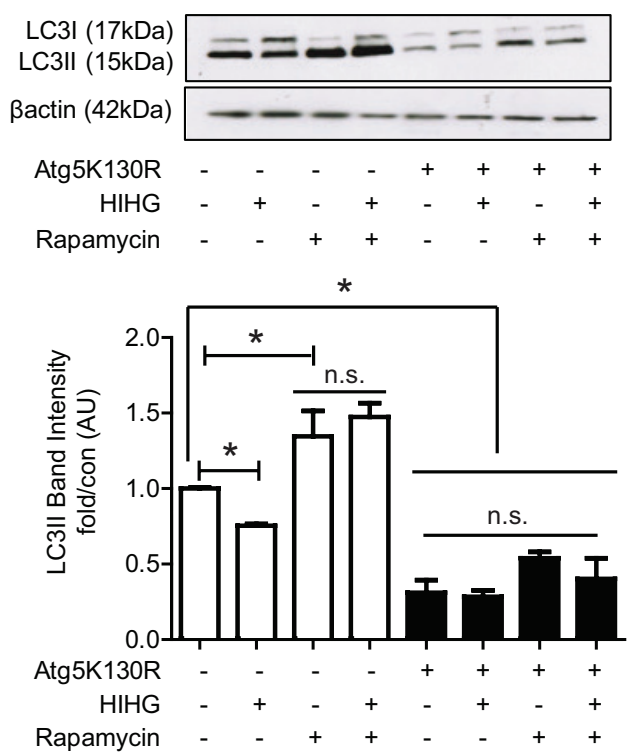
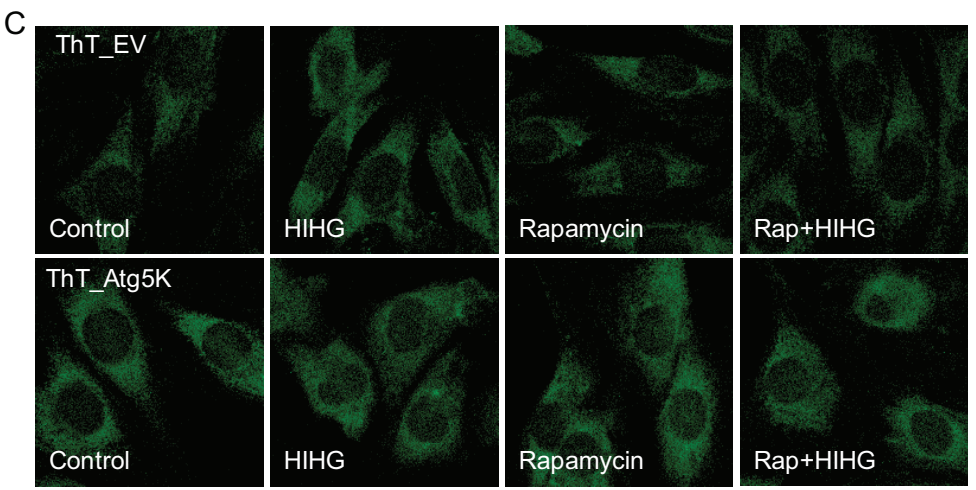

*

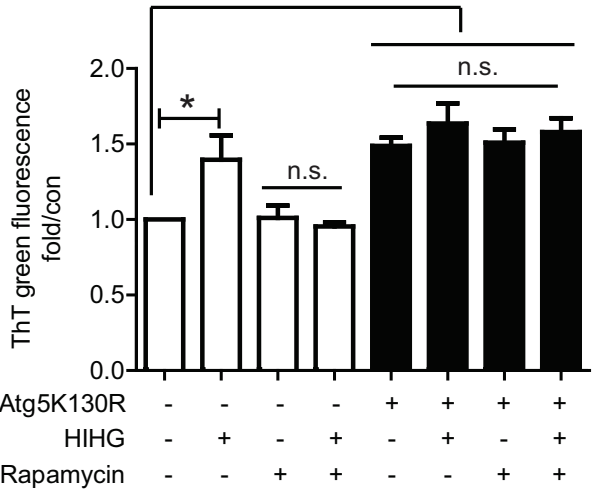

Figure 4

Rapamycin treatment increased autophagy and decreased HIHG-induced ER stress in EV but not Atg5K cells. (A) Quantitative and statistical analysis of red-to-green fluorescence ratio in tandem fluorescence-GFP-RFP-LC3 L6 cells in HIHG condition compared to control, with or without rapamycin pre-treatment, $n=4$; (B) Representative images and densitometry analysis of LC3 in response to rapamycin pre-treatment in HIHG-treated EV and Atg5K cells, $n=3$; (C) Representative confocal images and quantitative and statistical analysis of ThT green fluorescence comparing the effect of rapamycin pre-treatment in HIHG condition in EV and Atg5K cells respectively, $n=4$. Values are mean \pm s.E.M., $* P<0.05$. A full color version of this figure is available at http://dx.doi.org/10.1530/JME-17-0096.

ER stress as indicated by significantly decreased ThT fluorescence in HIHG and adiponectin treatment compared to HIHG alone (Fig. 6D). To test whether adiponectin restored HIHG-impaired insulin signaling and induced ER stress was autophagy dependent or not, we used the autophagic deficient Atg5K130R cell line. Adiponectin maintained insulin sensitivity, measured by phosphorylation of AKT T308, in HIHG treated normal but not autophagy-deficient cells (Fig. 6E).

\section{Discussion}

Metabolic dysfunction, occurring at least in part via insulin resistance, in skeletal muscle plays a major role in the development of the metabolic syndrome and type 2 diabetes (Marette et al. 2014). New knowledge contributing to our understanding of the mechanisms responsible for development of skeletal muscle insulin resistance is thus of great importance in order to facilitate development of improved therapeutic approaches. Various mechanisms have been suggested to contribute to the pathophysiology of metabolic dysfunction, and our previous studies have helped to establish skeletal muscle as an important target tissue for the actions of adiponectin, an anti-diabetic hormone, which normally circulates at high levels, but which is reduced in obesity and diabetes (Liu \& Sweeney 2014, Marette et al. 2014, Stern et al. 2016). In skeletal muscle, adiponectin mediates direct metabolic effects as well as improved insulin sensitivity. The cellular signaling pathways via which adiponectin confers beneficial metabolic effects have been well characterized and many effects are mediated via an AdipoR/APPL1/AMPK

Published by Bioscientifica Ltd. 
A
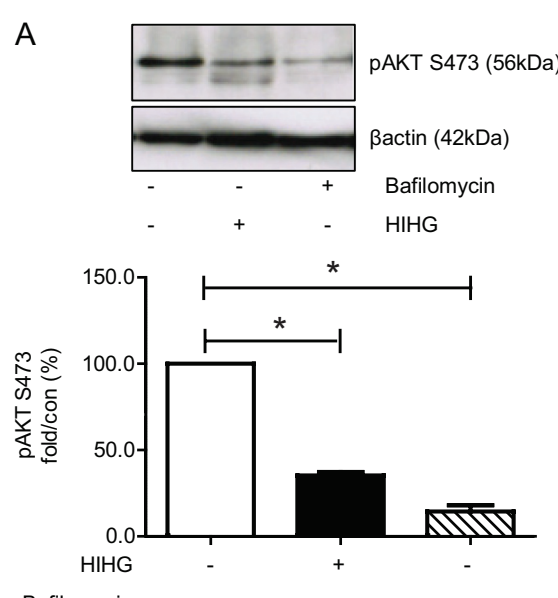
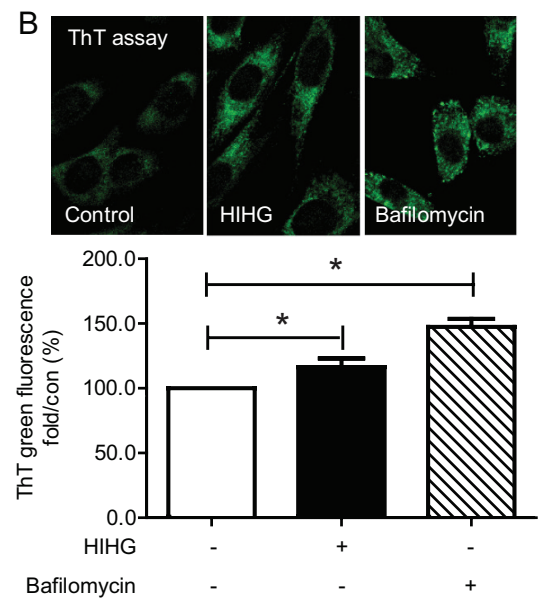
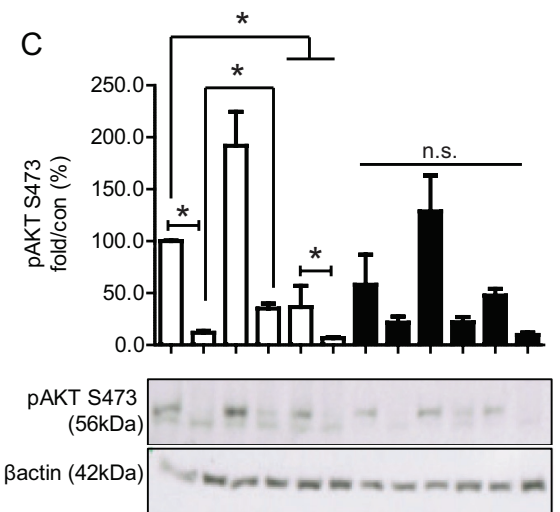

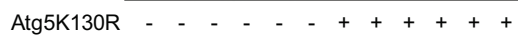

HIHG -+-+-++-++

Rapamycin - - + + - - - + + -

Bafilomycin - - - $-++\ldots$ - $-\quad++$

Bafilomycin

\section{Figure 5}

Inhibition of autophagy with bafilomycin treatment increased ER stress and decreased AKT phosphorylation. (A) Representative images and densitometry analysis of pAKT in BafA-treated cells compared to HIHG condition and control, $n=3$. (B) Representative confocal images and quantitative and statistical analysis of ThT green fluorescence in BafA-treated cells, $n=4$. (C) Representative images and densitometry analysis of insulin-stimulated PAKT in EV and Atg5K cells in response to BafA and rapamycin treatment, $n=3$. Values are mean \pm S.E.M., $* P<0.05$. A full color version of this figure is available at http://dx.doi.org/10.1530/JME-17-0096.

axis (Cheng et al. 2014). Indeed, it is expected that development of strategies to mimic adiponectin action can translate into effective therapeutic applications. For example, a small molecule adiponectin receptor agonist has been shown to activate AMPK via AdipoR's and ameliorate insulin resistance and glucose intolerance in $\mathrm{db} / \mathrm{db}$ mice or mice fed a high-fat diet (Okada-Iwabu et al. 2013). To study novel mechanisms via which adiponectin
A
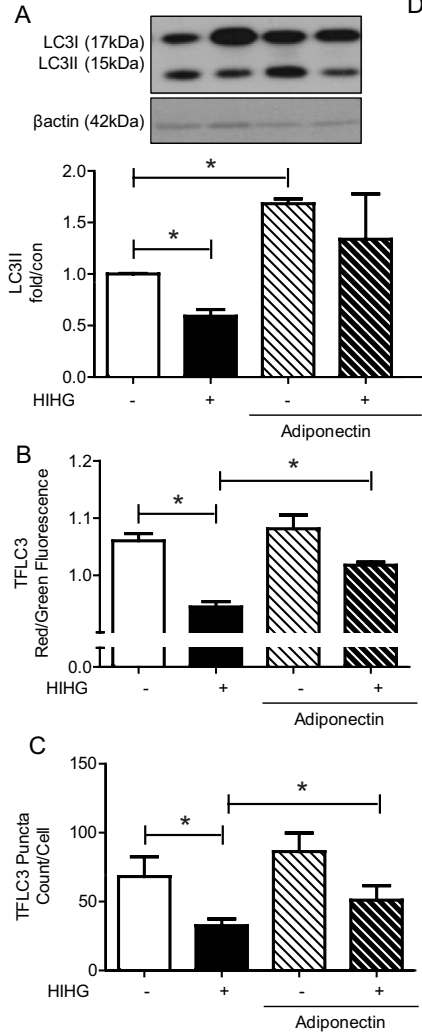
DOI: 10.1530/JME-17-0096
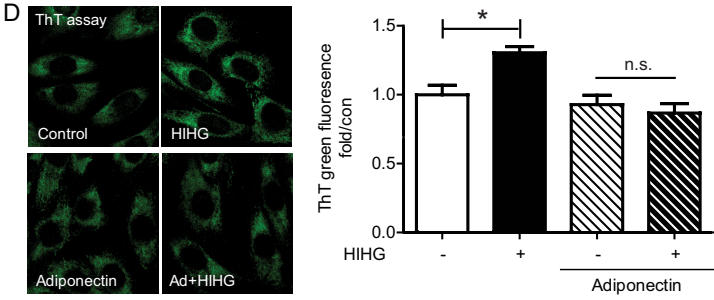

E pAKT T308 (56kDa) Bactin (42kDa)
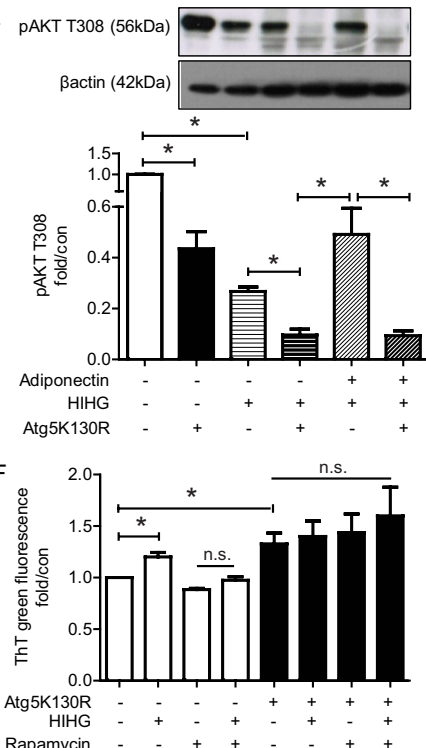

\section{Figure 6}

Adiponectin alleviates ER stress and improves insulin sensitivity in an autophagy-dependent manner. (A) Representative images and densitometry analysis of LC3 in HIHG condition with or without adiponectin pre-treatment, $n=3$; (B) Quantitative and statistical analysis of red-to-green fluorescence ratio in tandem fluorescence-GFP-RFP-LC3 L6 cells in HIHG condition compared to control, with or without adiponectin pre-treatment, $n=4$; (C) Quantitative analysis of TFLC3 puncta signal in HIHG condition compared to control, with or without adiponectin pre-treatment, $n=4$; (D) Representative confocal images and quantitative and statistical analysis of ThT green fluorescence in HIHG condition with or without adiponectin pre-treatment, $n=4$;

(E) Representative images and densitometry analysis of pAKT in HIHG condition comparing adiponectin pre-treatment in EV and Atg5K cells, $n=3$; (F) Quantitative and statistical analysis of ThT green fluorescence comparing the effect of adiponectin pre-treatment in HIHG condition in $\mathrm{EV}$ and Atg5K cells respectively, $n=4$. Values are mean \pm S.E.M., $* P<0.05$. A full color version of this figure is available at http://dx.doi.org/10.1530/ JME-17-0096. 
promoted insulin sensitivity, we used L6 skeletal muscle cells treated with HIHG to induce insulin resistance (Huang et al. 2002). Importantly, we first demonstrated that adiponectin treatment improved insulin signaling in this model, which is in keeping with previous work showing that adiponectin increased insulin sensitivity in other models (Liu et al. 2015).

We recently showed that one important cellular mechanism induced by adiponectin is autophagy (Jahng et al. 2015, Liu et al. 2015, Xu \& Sweeney 2015). Adiponectin directly stimulated autophagy in skeletal muscle cells and mice lacking adiponectin had reduced high-fat diet-induced changes in skeletal muscle autophagy (Liu et al. 2015). Nevertheless, we believe it is important to appreciate that alterations in autophagy under conditions regulating insulin sensitivity are likely to be highly time and tissue dependent. For example, in skeletal muscle 6 weeks of caloric restriction in diet-induced obese mice conferred beneficial metabolic effects in close correlation with changes in autophagy yet 16 weeks of high-fat diet induced only minor changes in markers of autophagy (Cui et al. 2013, Campbell et al. 2015). Conflicting data exist in adipose tissue, where 8 weeks of high-fat diet attenuated autophagy and 17 weeks of high-fat diet did not significantly alter autophagy (Zhou et al. 2016, Rocha-Rodrigues et al. 2017). Furthermore, adiponectin or pharmacological strategies to boost autophagy improved insulin sensitivity but adiponectinstimulated improvements in insulin sensitivity were lost in autophagy-deficient muscle cells (Liu et al. 2015). Here we show that adiponectin was able to counteract the decrease in autophagy which occurred in response to HIHG treatment of L6 cells. Our observations are in keeping with a growing body of recent studies which have shown that promoting autophagy by various approaches is beneficial in terms of improving insulin sensitivity (Wang et al. 2015, Zhu et al. 2016, Ghareghani et al. 2017, Li et al. 2017, Rosa-Caldwell et al. 2017). For example, using a model of palmitate-induced insulin resistance, it was shown that stress-inducible protein Sestrin2 (Sesn2) up-regulation maintained insulin sensitivity and glucose metabolism via AMPK-dependent autophagic activation (Li et al. 2017). It is also of interest to note other data which support the general dogma that autophagy positively correlates with insulin sensitivity. In skeletal muscle from severely insulin-resistant patients with T2D, there was decreased expression of autophagy-related gene (ATG14, RB1CC1/FIP200, GABARAPL1, SQSTM1/p62, and WIPI1) and protein (LC3BII, SQSTM1/p62 and ATG5) (Moller et al. 2017). Furthermore, in aged mice, there also was a decline in LC3B-I conjugation to phosphatidylethanolamine (PE), possibly due to decreased protein levels of ATG3 and ATG12-ATG5 (Zhou et al. 2017). We then speculated that crosstalk occurred between autophagy, induced by adiponectin, and the UPR and next investigated this.

Numerous studies have now clearly established that induction of ER stress in skeletal muscle can elicit insulin resistance (Quan et al. 2015, Dai et al. 2016, Kwak et al. 2016, Liong \& Lappas 2016). For example, tunicamycin or palmitate stimulated ER stress and insulin resistance while suppression of ER stress, using ER stress inhibitor tauroursodeoxycholic acid (TUDCA), melatonin or siRNA knockdown of IRE1 $\alpha$ and GRP78, significantly downregulated insulin resistance in human skeletal muscle (Quan et al. 2015, Dai et al. 2016, Liong \& Lappas 2016). The signaling protein tribbles 3 was shown to mediate endoplasmic reticulum stress-induced insulin resistance in skeletal muscle (Koh et al. 2013). Skeletal muscle kidney-enriched inositol polyphosphate phosphatase (SKIP), a PIP3 (phosphatidylinositol-3,4,5trisphosphate) phosphatase, has also been proposed to link ER stress to insulin resistance in skeletal muscle (Ijuin et al. 2016). Here, we have shown that HIHG induced ER stress. Difficulties with directly measuring ER stress has often necessitated investigators measuring UPR activation as an indication of ER stress, which can be misleading, and so here we used thioflavin $\mathrm{T}$ to detect protein aggregates (Beriault \& Werstuck 2013) and colocalized this signal with KDEL immunofluorescence to localize ER. Our data showed that HIHG enhanced ER-localized protein aggregates. We also found that HIHG-induced the expression of a GRP78 promoter-dependent fluorescent reporter and pIRE1, pPERK and ATF6. ER stress induced induction of activating transcription factor 6 (ATF6) and $\mathrm{X}$-box-binding protein 1 (XBP1)-dependent expression of SKIP was previously shown to decrease insulin sensitivity (Ijuin et al. 2016), and this is in keeping with the elevated ATF6, which we observed.

Most importantly, a phenomenon which is clearly emerging from recent studies is that activating autophagy can attenuate ER stress-induced decreases in insulin sensitivity in various tissues (Hwang et al. 2013, Wang et al. 2015, Kwak et al. 2016). An important role for AMPK in this process is apparent. We showed that adiponectinstimulated autophagy in muscle cells occurred via an AMPK-dependent signaling mechanism (Liu et al. 2015). Huang and coworkers showed that decreased insulinstimulated signaling and glucose uptake due to ER stress was rescued with the specific ERK inhibitor U0126 via

Published by Bioscientifica Ltd. 

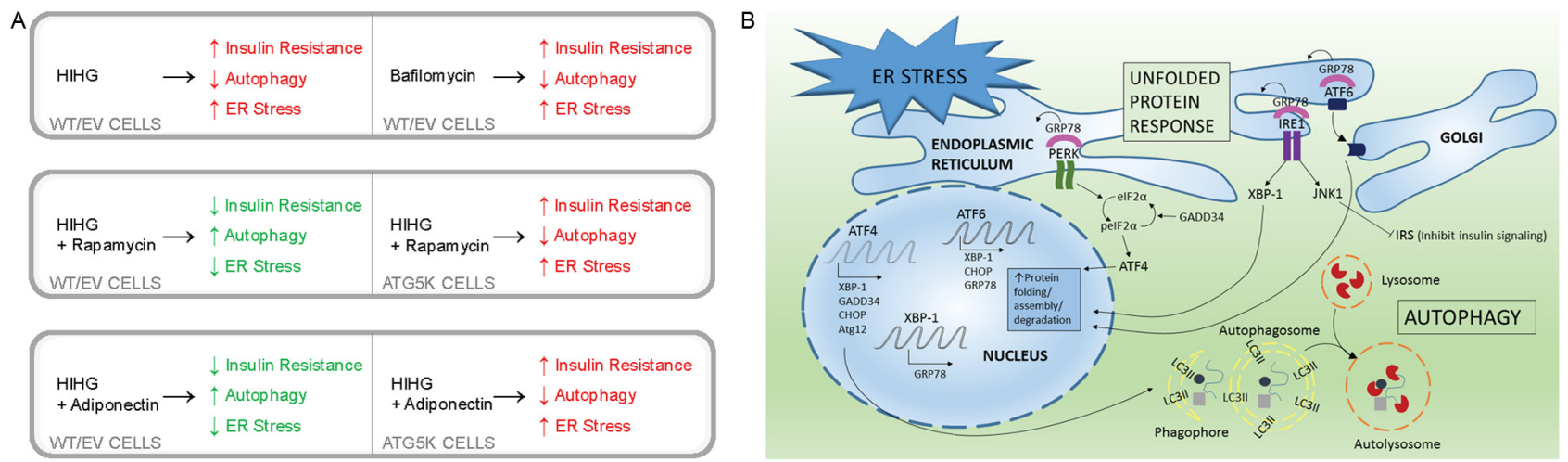

\section{Figure 7}

Schematic representation of conclusions. (A) Summary of main findings of this study in wild type/empty vector (WT/EV) or autophagy-deficient (Atg5K) cells. (B) Depiction of potential crosstalk between ER stress, the UPR, autophagy and insulin signaling. Briefly, disruption of normal ER function leads to an accumulation of unfolded protein. Increasing ER stress causes activation of the UPR. There are three branches of the UPR controlled by three ER transmembrane proteins: PERK, IRE1 and ATF6. In normal conditions, the ER chaperone GRP78 is bound to their ER luminal domain maintaining them in an inactive state; however, the increased levels of unfolded proteins in ER stress conditions cause GRP78 to dissociate. This allows their activation (phosphorylation of PERK and IRE1 and cleavage of ATF6) and initiation of their respective downstream signaling cascades. The function of these signaling cascades is to increase the capacity for protein folding and assembly but also to decrease the protein folding load by increasing ER associated degradation of unfolded proteins. In keeping witht his, it has been suggested that the UPR potentially induces autophagy via activation of elf2 $\alpha$ downstream of PERK, such that unfolded proteins associated with ER stress are degraded also by autophagy. In our study, we showed that disruption of autophagy caused ER stress and insulin resistance and that by restoring autophagy there was reduced ER stress and improved insulin sensitivity. Further, we demonstrated a decrease in pelf $2 \alpha$ after HIHG treatment, potentially suggesting that chronically elevated ER stress leads to a downregulation of UPR-initiated autophagy. Future studies to further examine the link between the UPR and induction of autophagy would yield insight into the role this mechanism plays in skeletal muscle insulin resistance in this cellular model. A full color version of this figure is available at http://dx.doi.org/10.1530/JME-17-0096.

AMPK activation (Huang et al. 2002). Furthermore, in $\mathrm{db} / \mathrm{db}$ mice, U0126 administration increased AMPK phosphorylation and improved insulin sensitivity in muscle tissues (Hwang et al. 2013). Similarly, the in vivo administration of bortezomib to ob/ob mice reduced ER stress marker levels, increased AMPK phosphorylation and improved insulin sensitivity in skeletal muscle (Kwak et al. 2016). In this study, stimulating autophagy with adiponectin or rapamycin alleviated ER stress and insulin resistance in normal but not autophagy-deficient cells, demonstrating their beneficial effect on insulin sensitivity was autophagy dependent.

Interestingly, the downregulation of autophagy, which we observed in response to HIHG might in part be due to altered UPR response. While PERK phosphorylation was elevated, we observed a notable and unexpected downregulation of peIf $2 \alpha$, a downstream target of PERK. Based on this, future research should examine other inputs to regulation of peIf $2 \alpha$ and if they are altered in insulin-resistant skeletal muscle cells. An additional interpretation is that since autophagy plays an important role in clearing unfolded proteins and alleviating ER stress (Pallet et al. 2008, Wang et al. 2015, Dash et al. 2016), impairment in autophagy might directly contribute to the observed increase in ER stress. In keeping with this, we demonstrated that both pharmacological blocking of autophagy using bafilomycin as well as molecular impairment of autophagy in the Atg5K autophagydeficient cell line caused an increase in ER stress. This conforms to previous findings that demonstrated autophagy-deficient models to be more susceptible to developing ER stress and insulin resistance (Munch et al. 2014, Wang et al. 2015).

In conclusion, our study demonstrated that HIHGinduced ER stress, reduced autophagy and insulin sensitivity in L6 skeletal muscle cells. Adiponectin treatment alleviated ER stress and improved insulin sensitivity in an autophagy-dependent manner (Fig. 7). These data further demonstrate an important role of autophagy in promoting skeletal muscle insulin sensitivity, particularly in response to adiponectin in skeletal muscle. This work develops our understanding of the cellular crosstalk between autophagy and ER stress in regulating insulin resistance and metabolic dysfunction in skeletal muscle. Via new knowledge such as this, we can generate new and improved therapies for metabolic complications in type 2 diabetes.

\section{Declaration of interest}

The authors declare that there is no conflict of interest that could be perceived as prejudicing the impartiality of the research reported. 


\section{Funding}

This work was funded by an operating grant to GS from Canadian Institutes of Health Research (CIHR).

\section{Acknowledgements}

G S also acknowledged support from Heart \& Stroke Foundation of Ontario via a Career Investigator Award.

\section{References}

Beriault DR \& Werstuck GH 2013 Detection and quantification of endoplasmic reticulum stress in living cells using the fluorescent compound, Thioflavin T. Biochimica et Biophysica Acta 1833 2293-2301. (doi:10.1016/j.bbamcr.2013.05.020)

Campbell TL, Mitchell AS, McMillan EM, Bloemberg D, Pavlov D, Messa I, Mielke JG \& Quadrilatero J 2015 High-fat feeding does not induce an autophagic or apoptotic phenotype in female rat skeletal muscle. Experimental Biology and Medicine 240 657-668. (doi:10.1177/1535370214557223)

Cheng KK, Lam KS, Wang B \& Xu A 2014 Signaling mechanisms underlying the insulin-sensitizing effects of adiponectin. Best Practice and Research: Clinical Endocrinology and Metabolism 28 3-13. (doi:10.1016/j.beem.2013.06.006)

Cui M, Yu H, Wang J, Gao J \& Li J 2013 Chronic caloric restriction and exercise improve metabolic conditions of dietary-induced obese mice in autophagy correlated manner without involving AMPK. Journal of Diabetes Research 2013 852754. (doi:10.1155/2013/852754)

Dai F, Jiang T, Bao YY, Chen GJ, Chen L, Zhang Q \& Lu YX 2016 Fenofibrate improves high-fat diet-induced and palmitate-induced endoplasmic reticulum stress and inflammation in skeletal muscle. Life Sciences 157 158-167. (doi:10.1016/j.lfs.2016.06.008)

Dash S, Chava S, Aydin Y, Chandra PK, Ferraris P, Chen W, Balart LA, Wu T \& Garry RF 2016 Hepatitis C virus infection induces autophagy as a prosurvival mechanism to alleviate hepatic ER-stress response. Viruses 8 E150. (doi:10.3390/v8050150)

Ghareghani P, Shanaki M, Ahmadi S, Khoshdel AR, Rezvan N, Meshkani R, Delfan M \& Gorgani-Firuzjaee S 2017 Aerobic endurance training improves nonalcoholic fatty liver disease (NAFLD) features via miR-33 dependent autophagy induction in high fat diet fed mice. Obesity Research and Clinical Practice [in press]. (doi:10.1016/j.orcp.2017.01.004)

Huang C, Somwar R, Patel N, Niu W, Torok D \& Klip A 2002 Sustained exposure of L6 myotubes to high glucose and insulin decreases insulin-stimulated GLUT4 translocation but upregulates GLUT4 activity. Diabetes 51 2090-2098. (doi:10.2337/diabetes.51.7.2090)

Hwang SL, Jeong YT, Li X, Kim YD, Lu Y, Chang YC, Lee IK \& Chang HW 2013 Inhibitory cross-talk between the AMPK and ERK pathways mediates endoplasmic reticulum stress-induced insulin resistance in skeletal muscle. British Journal of Pharmacology 169 69-81. (doi:10.1111/bph.12124)

Ijuin T, Hosooka T \& Takenawa T 2016 Phosphatidylinositol 3,4,5-trisphosphate phosphatase SKIP links endoplasmic reticulum stress in skeletal muscle to insulin resistance. Molecular and Cellular Biology 36 108-118.

Jahng JW, Turdi S, Kovacevic V, Dadson K, Li RK \& Sweeney G 2015 Pressure overload-induced cardiac dysfunction in aged male adiponectin knockout mice is associated with autophagy deficiency. Endocrinology 156 2667-2677. (doi:10.1210/en.2015-1162)

Koh HJ, Toyoda T, Didesch MM, Lee MY, Sleeman MW, Kulkarni RN, Musi N, Hirshman MF \& Goodyear LJ 2013 Tribbles 3 mediates endoplasmic reticulum stress-induced insulin resistance in skeletal muscle. Nature Communications 4 1871. (doi:10.1038/ncomms2851)
Kwak HJ, Choi HE, Jang J, Park SK, Bae YA \& Cheon HG 2016 Bortezomib attenuates palmitic acid-induced ER stress, inflammation and insulin resistance in myotubes via AMPK dependent mechanism. Cell Signaling 28 788-797. (doi:10.1016/j.cellsig.2016.03.015)

Lai E, Bikopoulos G, Wheeler MB, Rozakis-Adcock M \& Volchuk A 2008 Differential activation of ER stress and apoptosis in response to chronically elevated free fatty acids in pancreatic beta-cells. American Journal of Physiology: Endocrinology and Metabolism 294 E540-E550. (doi:10.1152/ajpendo.00478.2007)

Li H, Liu S, Yuan H, Niu Y \& Fu L 2017 Sestrin 2 induces autophagy and attenuates insulin resistance by regulating AMPK signaling in C2C12 myotubes. Experimental Cell Research 354 18-24. (doi:10.1016/j. yexcr.2017.03.023)

Liong S \& Lappas M 2016 Endoplasmic reticulum stress regulates inflammation and insulin resistance in skeletal muscle from pregnant women. Molecular and Cellular Endocrinology 425 11-25. (doi:10.1016/j.mce.2016.02.016)

Liu Y \& Sweeney G 2014 Adiponectin action in skeletal muscle. Best Practice and Research: Clinical Endocrinology and Metabolism 28 33-41. (doi:10.1016/j.beem.2013.08.003)

Liu Y, Palanivel R, Rai E, Park M, Gabor TV, Scheid MP, Xu A \& Sweeney G 2015 Adiponectin stimulates autophagy and reduces oxidative stress to enhance insulin sensitivity during high-fat diet feeding in mice. Diabetes 64 36-48. (doi:10.2337/db14-0267)

Marette A, Liu Y \& Sweeney G 2014 Skeletal muscle glucose metabolism and inflammation in the development of the metabolic syndrome. Reviews in Endocrine and Metabolic Disorders 15 299-305. (doi:10.1007/s11154-014-9296-6)

Moller AB, Kampmann U, Hedegaard J, Thorsen K, Nordentoft I, Vendelbo MH, Moller N \& Jessen N 2017 Altered gene expression and repressed markers of autophagy in skeletal muscle of insulin resistant patients with type 2 diabetes. Scientific Reports 7 43775. (doi:10.1038/srep43775)

Munch D, Rodriguez E, Bressendorff S, Park OK, Hofius D \& Petersen M 2014 Autophagy deficiency leads to accumulation of ubiquitinated proteins, ER stress, and cell death in Arabidopsis. Autophagy 10 1579-1587. (doi:10.4161/auto.29406)

Okada-Iwabu M, Yamauchi T, Iwabu M, Honma T, Hamagami K, Matsuda K, Yamaguchi M, Tanabe H, Kimura-Someya T, Shirouzu M, et al. 2013 A small-molecule AdipoR agonist for type 2 diabetes and short life in obesity. Nature 503 493-499. (doi:10.1038/nature12656)

Palanivel R, Fang X, Park M, Eguchi M, Pallan S, De Girolamo S, Liu Y, Wang Y, Xu A \& Sweeney G 2007 Globular and full-length forms of adiponectin mediate specific changes in glucose and fatty acid uptake and metabolism in cardiomyocytes. Cardiovascular Research 75 148-157. (doi:10.1016/j.cardiores.2007.04.011)

Pallet N, Bouvier N, Legendre C, Gilleron J, Codogno P, Beaune P, Thervet E \& Anglicheau D 2008 Autophagy protects renal tubular cells against cyclosporine toxicity. Autophagy 4 783-791.

Quan X, Wang J, Liang C, Zheng H \& Zhang L 2015 Melatonin inhibits tunicamycin-induced endoplasmic reticulum stress and insulin resistance in skeletal muscle cells. Biochemical and Biophysical Research Communications 463 1102-1107. (doi:10.1016/j.bbrc.2015.06.065)

Rocha-Rodrigues S, Goncalves IO, Beleza J, Ascensao A \& Magalhaes J 2017 Effects of endurance training on autophagy and apoptotic signaling in visceral adipose tissue of prolonged high fat diet-fed rats. European Journal of Nutrition [in press]. (doi:10.1007/s00394-0171500-5)

Rosa-Caldwell ME, Lee DE, Brown JL, Brown LA, Perry RA Jr, Greene ES, Carvallo Chaigneau FR, Washington TA \& Greene NP 2017 Moderate physical activity promotes basal hepatic autophagy in diet-induced obese mice. Applied Physiology, Nutrition, and Metabolism 42 148-156. (doi:10.1139/apnm-2016-0280)

Salvado L, Barroso E, Gomez-Foix AM, Palomer X, Michalik L, Wahli W \& Vazquez-Carrera M 2014 PPARbeta/delta prevents endoplasmic reticulum stress-associated inflammation and insulin resistance in skeletal muscle cells through an AMPK-dependent 
mechanism. Diabetologia 57 2126-2135. (doi:10.1007/s00125014-3331-8)

Stern JH, Rutkowski JM \& Scherer PE 2016 Adiponectin, leptin, and fatty acids in the maintenance of metabolic homeostasis through adipose tissue crosstalk. Cell Metabolism 23 770-784. (doi:10.1016/j. cmet.2016.04.011)

Wang H, Sun RQ, Zeng XY, Zhou X, Li S, Jo E, Molero JC \& Ye JM 2015 Restoration of autophagy alleviates hepatic ER stress and impaired insulin signalling transduction in high fructose-fed male mice. Endocrinology 156 169-181. (doi:10.1210/en.2014-1454)

Xu A \& Sweeney G 2015 Emerging role of autophagy in mediating widespread actions of ADIPOQ/adiponectin. Autophagy 11 723-724. (doi:10.1080/15548627.2015.1034418)

Zhang F, Zhao S, Yan W, Xia Y, Chen X, Wang W, Zhang J, Gao C, Peng C, Yan F, et al. 2016 Branched chain amino acids cause liver injury in obese/diabetic mice by promoting adipocyte lipolysis and inhibiting hepatic autophagy. EBioMedicine 13 157-167. (doi:10.1016/j.ebiom.2016.10.013)

Zhou B, Li H, Liu J, Xu L, Guo Q, Zang W, Sun H \& Wu S 2016 Autophagic dysfunction is improved by intermittent administration of osteocalcin in obese mice. International Journal of Obesity $\mathbf{4 0}$ 833-843. (doi:10.1038/ijo.2016.1)

Zhou J, Chong SY, Lim A, Singh BK, Sinha RA, Salmon AB \& Yen PM 2017 Changes in macroautophagy, chaperone-mediated autophagy, and mitochondrial metabolism in murine skeletal and cardiac muscle during aging. Aging 9 583-599.

Zhu S, Wu Y, Ye X, Ma L, Qi J, Yu D, Wei Y, Lin G, Ren G \& Li D 2016 FGF21 ameliorates nonalcoholic fatty liver disease by inducing autophagy. Molecular and Cellular Biochemistry 420 107-119. (doi:10.1007/s11010-016-2774-2)

Received in final form 6 August 2017

Accepted 4 September 2017
Published by Bioscientifica Ltd. 